\title{
REVIEW
}

Open Access

\section{The role of mesenchymal stem cells in hematopoietic stem cell transplantation: prevention and treatment of graft-versus- host disease}

\author{
Lu Zhao ${ }^{1,2}$, Shanquan Chen ${ }^{3}$, Panxin Yang ${ }^{1,2}$, Hongcui Cao ${ }^{1,2^{*}}$ (i) and Lanjuan Li 1, $^{1,2}$
}

\begin{abstract}
Background: The use and effectiveness of hematopoietic stem cell transplantation (HSCT) are limited by lethal complications, i.e., acute and chronic graft-versus-host disease (aGVHD and cGVHD, respectively), in which immune cells from the donor attack healthy recipient tissues. GVHD presents both prophylactic and therapeutic challenges, and overall survival is poor. Mesenchymal stem cells (MSCs) show considerable promise in the treatment of GVHD because of their potential immunomodulatory activity. Multiple studies have been performed to explore the possible benefit of MSCs in GVHD, but the results of these studies are sometimes conflicting. Therefore, we performed a systematic review and meta-analysis to estimate the effect of MSC infusion on GVHD treatment and prevention.

Methods: We systematically searched the MEDLINE (PubMed), Cochrane Library, EMBASE, ClinicalTrials.gov, and SinoMed CBM databases to identify studies published before February 2018 involving patients with hematologic malignancies undergoing HSCT and receiving MSC-based or conventional therapy. We included studies if they reported on the outcomes of interest.

Results: Ultimately, 10 studies were selected from among 413 candidates. According to our meta-analyses, compared with conventional treatment, MSC therapy demonstrated substantial improvements in terms of complete response (CR) and overall survival for CGVHD. However, MSC therapy did not show substantial improvements in terms of engraftment, the incidence of aGVHD, relapse, death, death due to relapse, or death due to infection. Subgroup analyses showed that MSCs derived from the umbilical cord (U-MSCS) and MSC infusion after HSCT substantially improved engraftment and CGVHD incidence, whereas MSCs derived from bone marrow (B-MSCs) and MSC infusion before HSCT shows no improvement. In addition, B-MSCs and MSC infusion before HSCT tend to prolong engraftment time, as well as increase the rates of relapse and death.

(Continued on next page)
\end{abstract}

\footnotetext{
* Correspondence: hccao@zju.edu.cn

${ }^{1}$ State Key Laboratory for Diagnosis and Treatment of Infectious Diseases, the

First Affiliated Hospital, College of Medicine, Zhejiang University, 79

Qingchun Rd., Hangzhou City 310003, China

${ }^{2}$ Collaborative Innovation Center for Diagnosis and Treatment of Infectious

Diseases, 79 Qingchun Rd., Hangzhou City 310003, China

Full list of author information is available at the end of the article
}

(c) The Author(s). 2019 Open Access This article is distributed under the terms of the Creative Commons Attribution 4.0 International License (http://creativecommons.org/licenses/by/4.0/), which permits unrestricted use, distribution, and reproduction in any medium, provided you give appropriate credit to the original author(s) and the source, provide a link to the Creative Commons license, and indicate if changes were made. The Creative Commons Public Domain Dedication waiver (http://creativecommons.org/publicdomain/zero/1.0/) applies to the data made available in this article, unless otherwise stated. 


\begin{abstract}
(Continued from previous page)
Conclusions: MSC infusion can reduce cGVHD but not aGVHD incidence and showed a positive effect in patients who already had aGVHD. For GVHD prevention, the use of U-MSCS and MSC infusion after HSCT were optimal for reducing CGVHD incidence and promoting engraftment, and might help decrease the incidence rate of relapse and death. However, B-MSCs and MSC infusion before HSCT may be harmful to patients and thus require serious consideration. A lack of robust evidence, owing to the small number of studies and small sample sizes, indicates a need for further high-quality clinical trials including large numbers of patients to validate our findings.
\end{abstract}

Keywords: Mesenchymal stem cells, Stem cell transplantation, Graft-versus-host disease, Meta-analysis

\section{Background}

Hematopoietic stem cell transplantation (HSCT) is an intensive therapy used to treat hematologic malignant disorders and genetic diseases. Modern approaches to human leukocyte antigen-haploidentical blood or marrow transplantation [1], and improvements of transplantation outcomes, have led to greater use of HSCTs. The number of HSCT procedures continues to increase, with more than 60,000 performed annually according to the Center for International Blood and Marrow Transplant Research [2]. However, its major lethal complication, graft-versus-host disease (GVHD), which may manifest as acute GVHD (aGVHD) or chronic GVHD (cGVHD), limits the effectiveness of HSCT [3].

GVHD is an immunological disorder in which immune cells from the donor attack healthy recipient tissues, including the gastrointestinal tract, liver, skin, and lungs. GVHD occurs in more than $50 \%$ of patients undergoing HSCT [4]. According to the extent of involvement of the affected organs, aGVHD is categorized into four types: I (mild), II (moderate), III (severe), and IV (very severe), while cGVHD is subdivided into limited cGVHD and extensive cGVHD. Given the current trend, the number of transplants from unrelated donors is expected to double within the next 5 years and will substantially increase the number of patients with GVHD. The threat posed by GVHD to patient survival is also gradually increasing.

GVHD presents both prophylactic and therapeutic challenges. Prophylactically, pharmacological manipulation of $\mathrm{T}$ cells after transplantation is the most commonly used preventive strategy. Administration of antibodies against T cells in vivo for GVHD prevention has been tested extensively using antithymocyte globulin (ATG) and antilymphocyte globulin (ALG) preparations. Unfortunately, neither drug is optimal for enhancing long-term survival, despite reducing the frequency of GVHD. Therapeutically, a steroid regimen, given their potent antilymphocyte and anti-inflammatory effects, remains the gold standard for treatment of GVHD [5]. However, less than half of patients with GVHD who underwent treatment with steroids achieved complete remission; on the contrary, more patients became steroid-refractory and showed very poor overall survival [6-8]. Despite important advances in the field of HSCT over the past few years, there has been little improvement in the morbidity or mortality of GVHD [9]. Patients with severe GVHD have a dismal estimated longterm survival rates, of 25\% (5 years) for grade III and 5\% for grade IV [10]. Allogeneic transplantation is becoming an increasingly attractive therapeutic option, thus accelerating the search for novel approaches to GVHD.

A cell-based therapeutic approach, using mesenchymal stem cells (MSCs), has recently shown considerable promise because of their expected immunomodulatory effects $[11,12]$. MSCs are plate-adhering, fibroblast-like cells known for their self-renewal capacity and ability to differentiate into multiple mesenchymal cell lineages [13]. In addition to their differentiation potential, MSCs are immunosuppressive. Bartholomew et al. first demonstrated that MSC administration in vivo could prolong skin graft survival [14]. Many subsequent in vitro and in vivo studies have confirmed the immunomodulatory activity of MSCs, by showing that they can inhibit the proliferation and functions of T cells, B cells, dendritic cells, and natural killer cells [15-18]. Studies have also reported that MSCs play an active role in promoting facilitation of HSC engraftment following transplantation, because they are part of the HSC niche, wherein they support hematopoiesis $[19,20]$. Owing to the growing understanding of MSCs, they have become an exciting tool for treating prophylaxis and GVHD in the HSCT setting and have been approved for use in clinical trials as immunomodulators [21].

Since Le Blanc et al. first reported complete remission of steroid-resistant aGVHD in a child receiving MSC infusions [22], multiple studies have been performed to explore the possible benefit of MSCs in GVHD. However, results are conflicting as to whether MSC infusion during HSCT is effective in managing GVHD [23]. Canada, New Zealand, and some EU countries have approved Prochymal ${ }^{\circ}$, the first MSC drug released to the market, for the treatment of children with steroidrefractory GVHD, while other countries including the USA and China have not. The field of MSC therapy is faced with a paradox regarding the clinical utility of MSCs for GVHD, with opposite clinical outcomes in the 
USA and Europe [23]. A previous meta-analysis [2] of uncontrolled studies with single-arm design was published in 2016 and showed that MSC treatment had a positive effect on 6-month survival in patients with aGVHD. However, no pooled analysis based on controlled trials has confirmed this report. The efficacy of MSC infusion for GVHD prevention is also controversial, with research variously showing both a significant benefit [24] and no benefit [25]. As evidence accumulates, it is essential to explore whether use of MSCs is favorable for GVHD, which represents the first area of clinical application of MSCs. Therefore, we performed a systematic review and meta-analysis of controlled trials to determine the effect of MSC infusion for both GVHD treatment and prevention.

\section{Methods}

\section{Search strategy and selection criteria}

We searched the Medline (PubMed) [26], Cochrane Library [27], EMBASE [28], ClinicalTrials.gov [29], and SinoMed CBM [30] databases up to February 2018 to identify relevant studies using a combined free text and $\mathrm{MeSH}$ heading search strategy (see Additional file 1), with no language or time restrictions. The retrieval strategy was based on the patient-intervention-comparisonoutcome (PICO) principle and was enhanced by adding keywords related to GVHD ("graft versus host disease", "graft vs. host disease" and "GVHD") and mesenchymal stem cells ("mesenchymal stem cell"," "mesenchymal stromal cell"*", and "multipotent stromal cell"*). We also checked the reference lists of the retrieved studies for additional relevant studies. The inclusion criteria were (1) randomized controlled trials (RCTs) for GVHD prevention, (2) controlled trials for GVHD treatment, (3) inclusion of patients who underwent HSCT, (4) use of MSC, and (5) availability of treatment outcome parameters [complete response $(\mathrm{CR})$ and overall survival] or prevention-related data [engraftment, aGVHD, cGVHD, relapse, death, death due to relapse, and death due to infection]. Studies were excluded if they were animalbased, review articles, or case reports. When duplicate reports from the same study were identified, the one including more information or a longer follow-up period was selected.

\section{Data extraction and statistical analysis}

For each study, data were extracted by one investigator and reviewed by a second investigator to ensure accuracy. Information on the following was extracted: patients (number, age, sex, disease information), MSCs (number, source, dose, number of infusions, and infusion timing), outcome parameters during the follow-up period, and study information (author, publication year, country, study design, and follow-up period).
A meta-analysis was conducted to evaluate whether the efficacy of MSC-based therapy was greater than that of conventional therapy in terms of GVHD prevention and treatment. Outcome parameters were evaluated by calculating the risk ratio (RR) or standardized mean difference (SMD) with 95\% confidence intervals (CIs). The percentage of variability across studies attributable to heterogeneity beyond chance was assessed using the chi-square-based $Q$ test $(P<0.1$ was considered indicative of significance) and $I^{2}$ statistic $\left(I^{2}>50 \%\right.$ indicated high heterogeneity). A forest plot was used to visualize the RR and 95\% CI for each study. A random-effects model was used because it provides a more conservative estimate of the presence of heterogeneity. A sensitivity analysis, with omission of one study at a time, was conducted to assess heterogeneity. Where sufficient studies were available, publication bias was assessed by the Egger test and visualized using Begg funnel plots [31]. Subgroup meta-analyses were conducted based on MSC source [bone marrow (B-MSCs) or umbilical cord (U-MSCs)] and MSC infusion timing (before HSCT or after HSCT), to identify factors related to the therapeutic efficacy of MSCs. All analyses were conducted using $\mathrm{R}$ software (version 3.4.0; $\mathrm{R}$ Development Core Team, Vienna, Austria).

\section{Results \\ Study selection}

As shown in Fig. 1, a total of 413 potentially eligible articles were identified by searching the five databases and the reference lists of the retrieved studies. Of these, 53 duplicate articles were excluded. After reading the titles, a further 152 articles were excluded (52 irrelevant papers, 36 animal experiments, 46 reviews and 18 case reports). After reading the abstracts, 181 additional articles were excluded (31 irrelevant papers, 12 animal experiments, 59 reviews, 71 uncontrolled trials, and 8 case reports). Among the remaining 27 articles, 23 concerned prevention and 4 concerned treatment. After assessing the complete texts, 16 of the 23 prevention-related articles were excluded because they were non-randomized controlled trials (nRCTs). The four treatment-related articles included two RCTs and two nRCTs. The two RCTs were meeting abstracts and one [32] of them was excluded because outcome parameters could not be extracted (i.e., survival rate was not reported, and the exact number of cases showing a CR was not available). The remaining three articles were included regardless of whether or not they were RCTs, as controlled trials related to treatment were limited. Thus, seven RCTs $[9,24,25,33-36]$ on prevention and three on treatment (two nRCTs [37, 38] and one RCT [39]) were finally analyzed. 


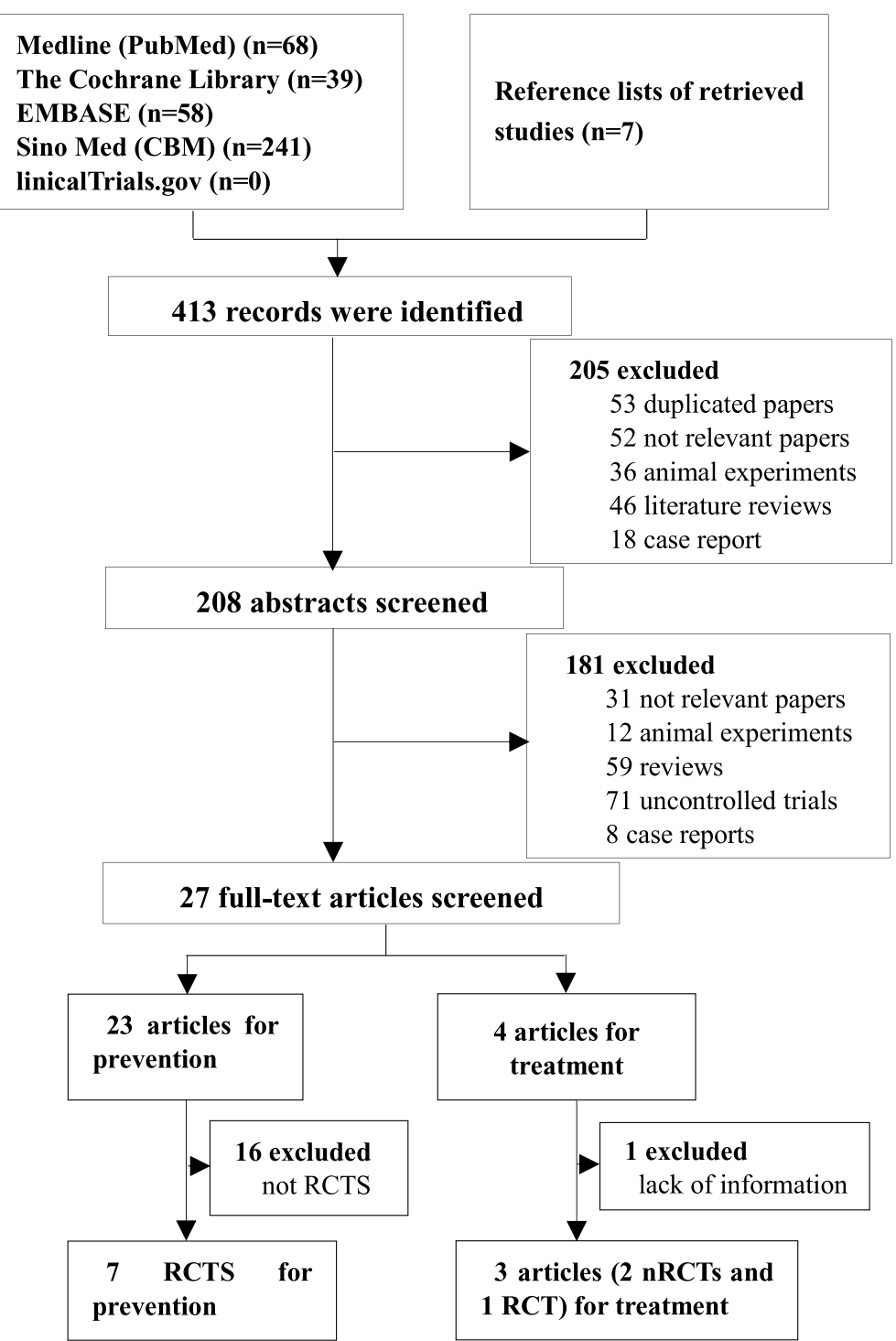

Fig. 1 Flowchart of study selection

\section{Characteristics of the included studies}

The characteristics of all 10 studies are presented in Tables 1 and 2. The studies were published between 2008 and 2017 and were conducted in China, Russia, Italy, and Sweden. The sample size ranged from 20 to 124 participants with malignant hematological diseases. Seven studies for prevention included a total of 402 patients and three studies for treatment included a total of 103 patients. All patients were treated with HSCT with or without an MSC infusion. The MSCs were B-MSCs or U-MSCs. The dose of infused MSCs ranged from $3.4 \times 10^{5}$ to $7.2 \times 10^{6}$ per kilogram. Infusions were only administered once for prevention, except in one study [9] wherein patients received multiple infusions. Infusions were administered multiple times for treatment, except for one [38] that did not report these data. MSCs were infused before [24, 36] or after [9, 25, 33-35] HSCT to prevent GVHD. For treatment, the median duration of aGVHD before MSC infusion ranged from 8 to 20 days. The follow-up period ranged from 12 to 70 months for prevention and 139 to 1312 days for treatment.

\section{Meta-analysis for treatment}

Among three articles, a total of 103 patients were suffering from aGVHD, 57 of whom underwent conventional treatment (control group); the remaining 46 patients received additional MSC infusions (MSC group).

\section{Complete response}

Two studies reported the number of patients with aGVHD who showed a CR in both MSC and control groups. Compared with the control group, patients in 


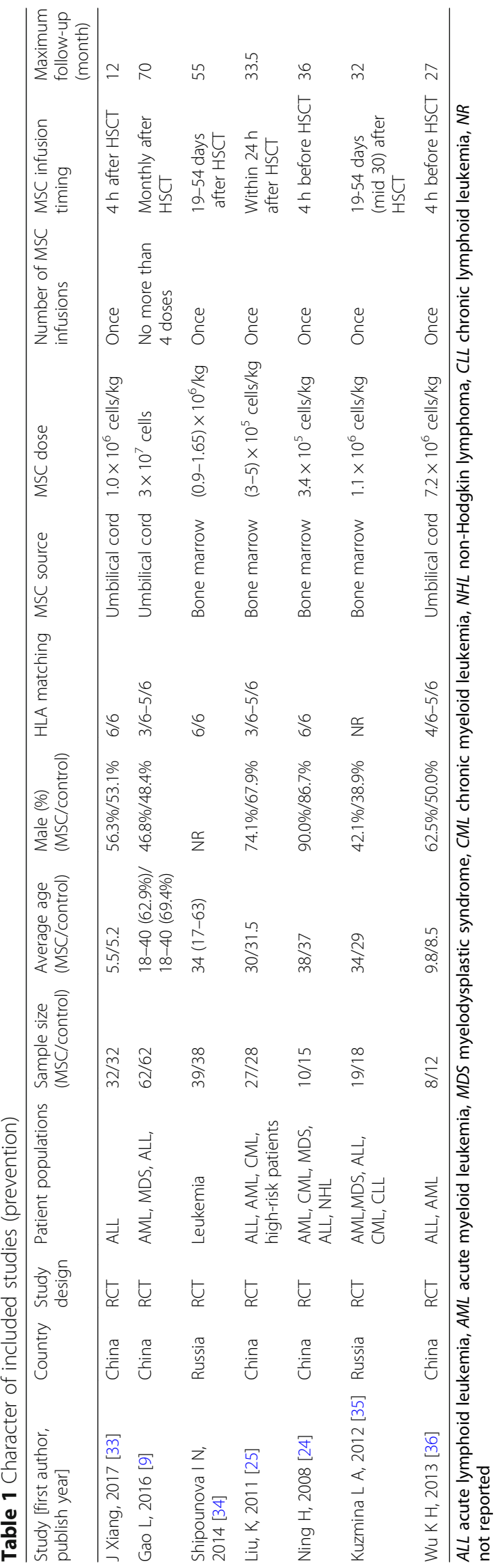




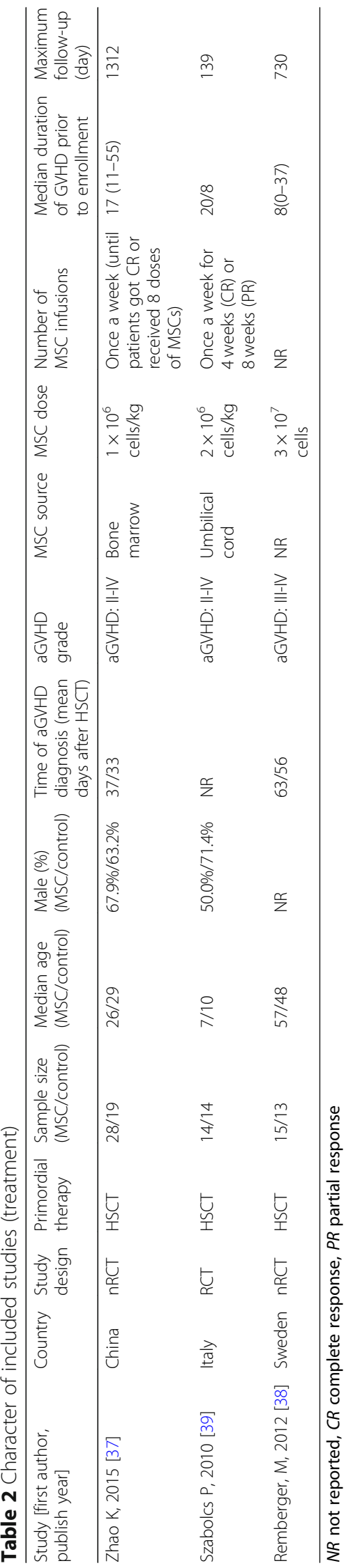


the MSC group had a significantly higher rate of CR $\left(\mathrm{RR}=2.28 ; 95 \%\right.$ CI: $1.24,4.18 ; I^{2}=0 \%, P=0.97$; Fig. 2$)$.

\section{Overall survival}

Three studies reported the number of patients with aGVHD who were alive during the follow-up in both the MSC and control groups. Compared with the control group, patients in the MSC group had a significantly higher rate of survival $\left(\mathrm{RR}=1.93 ; 95 \% \mathrm{CI} 1.12,3.32 ; I^{2}=\right.$ $41 \%, P=0.18$; Fig. 2).

\section{Meta-analysis for prevention}

Among seven RCTs on GVHD prevention, a total of 402 patients with hematologic malignancies underwent HSCT, of whom 205 were undergoing conventional GVHD prevention (control group); the remaining 197 patients received additional MSC infusions (MSC group)

\section{Engraftment}

Three studies reported the mean time to neutrophil engraftment (absolute neutrophil count $>0.5 \times 10^{9} / \mathrm{L}$ ) in both the MSC and control groups. Compared with the control group, patients in the MSC group had a shorter time to neutrophil engraftment, but the difference was not statistically significant $(\mathrm{SMD}=-1.20 ; 95 \% \mathrm{CI}-2.57,0.17$; $\left.I^{2}=88 \%, P<0.01\right)$. Significant heterogeneity existed and sensitivity analyses showed that the study of Ning [24] had the largest effect on the heterogeneity. Excluding this study decreased the heterogeneity to a non-significant level $\left(\mathrm{SMD}=-1.89 ; 95 \%\right.$ CI $\left.-2.42,-1.37 ; I^{2}=0 \%, P=0.91\right)$. The heterogeneity was likely related to the different sources used of MSCs. According to a subgroup analysis based on the source of MSCs (B-MSCs or U-MSCs), the U-MSC subgroup ( $\mathrm{SMD}=-1.89 ; 95 \% \mathrm{CI}-2.42,-1.37)$ showed a significantly shorter time to neutrophil engraftment in the MSC group compared with the control group, whereas the B-MSC subgroup ( $\mathrm{SMD}=0.13 ; 95 \% \mathrm{CI}-0.67,0.93$ ) showed a longer (but not statistically significant) time to neutrophil engraftment. According to a subgroup analysis based on MSC infusion time (before or after HSCT), the after subgroup $(\mathrm{SMD}=-1.91 ; 95 \% \mathrm{CI}-2.51,-1.31)$ showed a significantly shorter latency to neutrophil engraftment compared with the control group, whereas the before subgroup ( $\mathrm{SMD}=-0.82 ; 95 \% \mathrm{CI}-2.74,1.10)$ showed a shorter but not statistically significant time to neutrophil engraftment. These results are shown in Fig. 3.

\section{Acute GVHD}

Five studies reported the number of patients who developed aGVHD within 100 days after HSCT in both the MSC and control groups. Compared with the control group, patients in the MSC group had a lower risk of aGVHD, but the difference was not statistically significant (RR $=0.59 ; 95 \%$ CI $0.34,1.03 ; I^{2}=39 \%, P=0.16$ ). According to a subgroup analysis based on the source of MSC, both the B-MSC (RR $=0.46 ; 95 \%$ CI $0.19,1.11)$ and U-MSC (RR $=0.75 ; 95 \%$ CI $0.34,1.67)$ subgroups showed a non-significantly lower risk of aGVHD compared with the control group. A subgroup analysis based on MSC infusion time also showed that both the before $(\mathrm{RR}=0.47 ; 95 \% \mathrm{CI} 0.11,2.02)$ and after $(\mathrm{RR}=0.57 ; 95 \%$ CI $0.25,1.30$ ) subgroups had a non-significantly lower risk of aGVHD compared with the control group. These results are shown in Fig. 3.

\section{Chronic GVHD}

Six studies reported the number of patients who developed cGVHD in both the MSC and control groups. Compared with the control group, patients in the

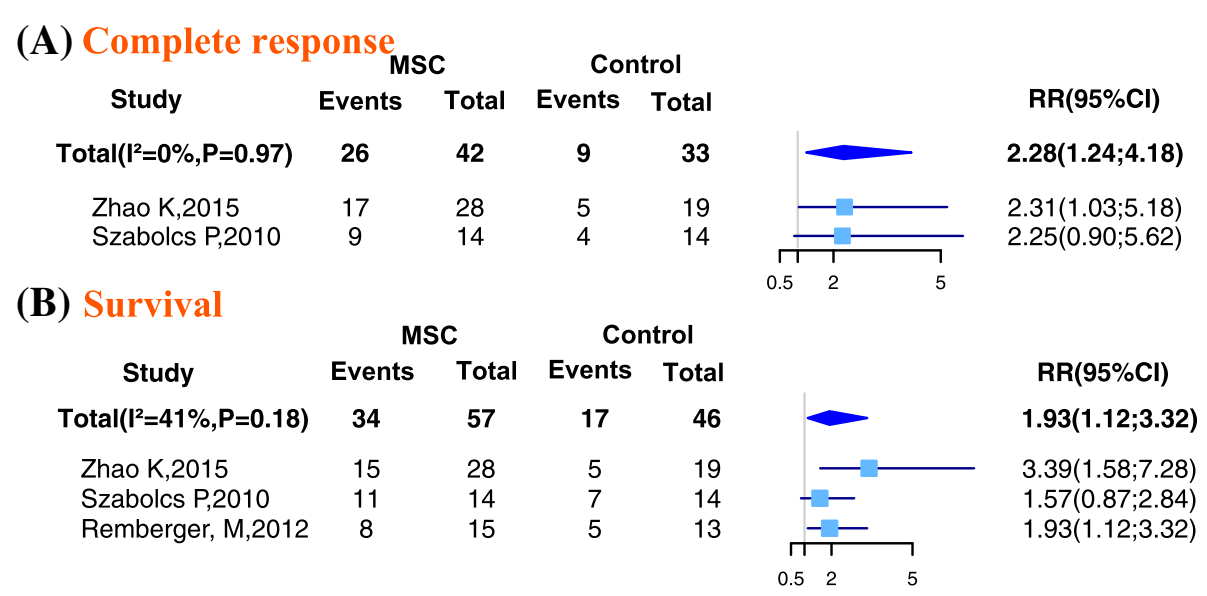

Fig. 2 Forest plot of graft-versus-host disease (GVHD) treatment. Compared with the control group, a the rate of complete response and $\mathbf{b}$ the rate of overall survival were significantly higher in the mesenchymal stem cell (MSC) group. Subgroup analyses could not be conducted because of an insufficient number of studies 


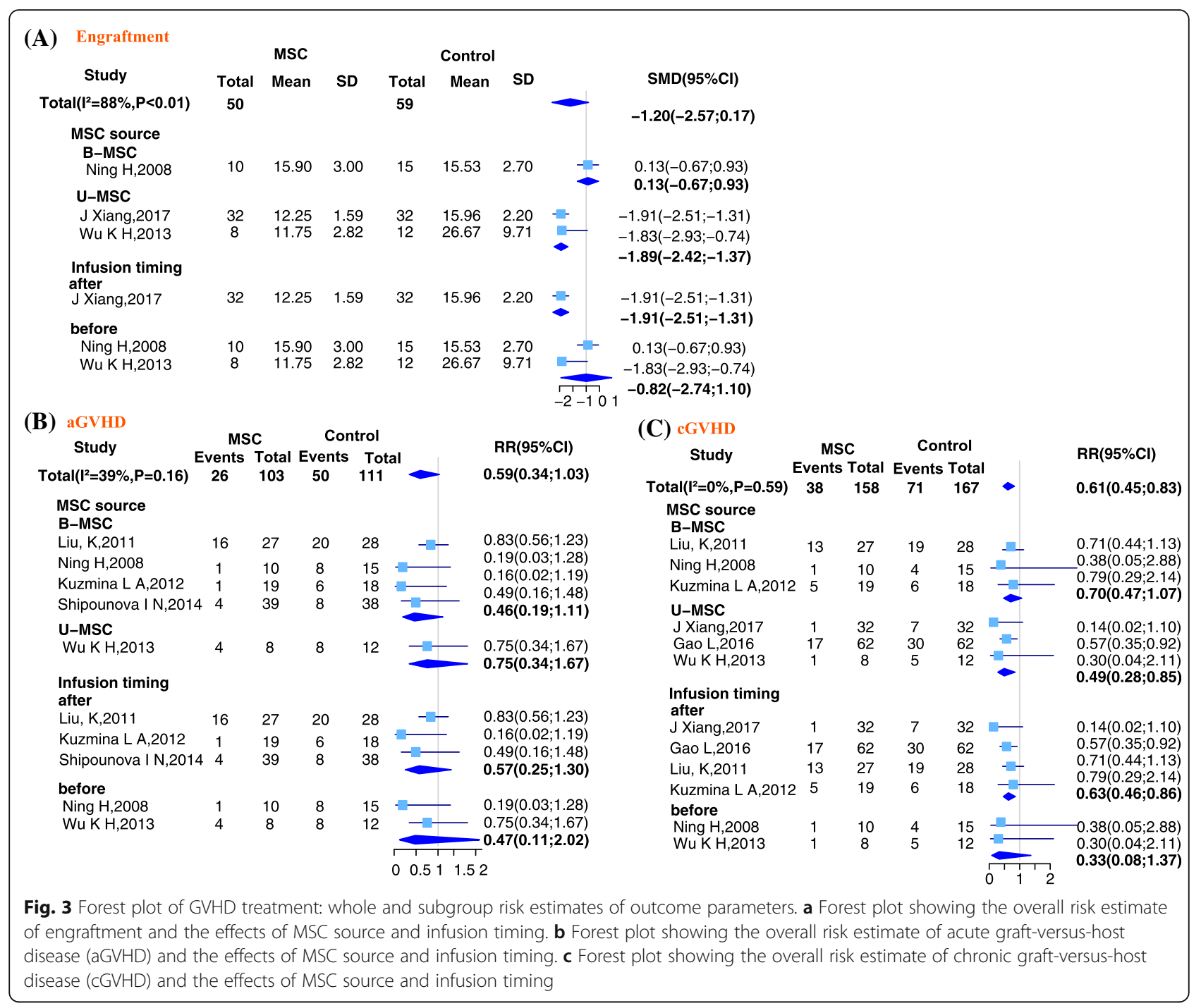

MSC group had a significantly lower risk of cGVHD $\left(\mathrm{RR}=0.61 ; 95 \%\right.$ CI $\left.0.45,0.83 ; I^{2}=0 \%, P=0.59\right)$. According to a subgroup analysis based on the source of MSC, only the U-MSC subgroup ( $R R=0.49 ; 95 \%$ CI $0.28,0.85$ ) showed a significantly lower risk of cGVHD compared with the control group; the BMSC subgroup ( $R R=0.70 ; 95 \%$ CI $0.47,1.07$ ) showed a non-significantly lower risk of cGVHD. According to a subgroup analysis based on MSC infusion time, only the after subgroup $(R R=0.63 ; 95 \%$ CI $0.46,0.86)$ showed a significantly lower risk of cGVHD compared with the control group; the before subgroup $(\mathrm{RR}=0.33 ; 95 \%$ CI $0.08,1.37)$ showed a nonsignificantly lower risk of cGVHD. These results are shown in Fig. 3.

\section{Relapse}

Seven studies reported the number of patients who had relapsed to the original malignant status after
HSCT in both the MSC and control group. Compared with the control groups, patients in the MSC group had a lower risk of relapse, but the difference was not statistically significant $(\mathrm{RR}=0.98 ; 95 \% \mathrm{CI}$ $0.70,1.39 ; I^{2}=0 \%, P=0.46$ ). According to a subgroup analysis based on the source of MSC, the U-MSC subgroup ( $R R=0.90 ; 95 \%$ CI $0.581,1.41)$ showed a lower (but not statistically significant) risk of relapse compared with the control group, whereas the BMSC subgroup ( $R R=1.20$; 95\% CI 0.59, 2.41) showed a higher (but not statistically significant) risk of relapse. According to subgroup analysis based on MSC infusion time, the after subgroup $(\mathrm{RR}=0.86 ; 95 \% \mathrm{CI}$ $0.59,1.24$ ) showed a lower (but not statistically significant) risk of relapse compared with the control group, whereas the before subgroup ( $R R=2.44 ; 95 \%$ CI $0.95,6.31$ ) showed a higher (but not statistically significant) risk of relapse. These results are shown in Fig. 4. 


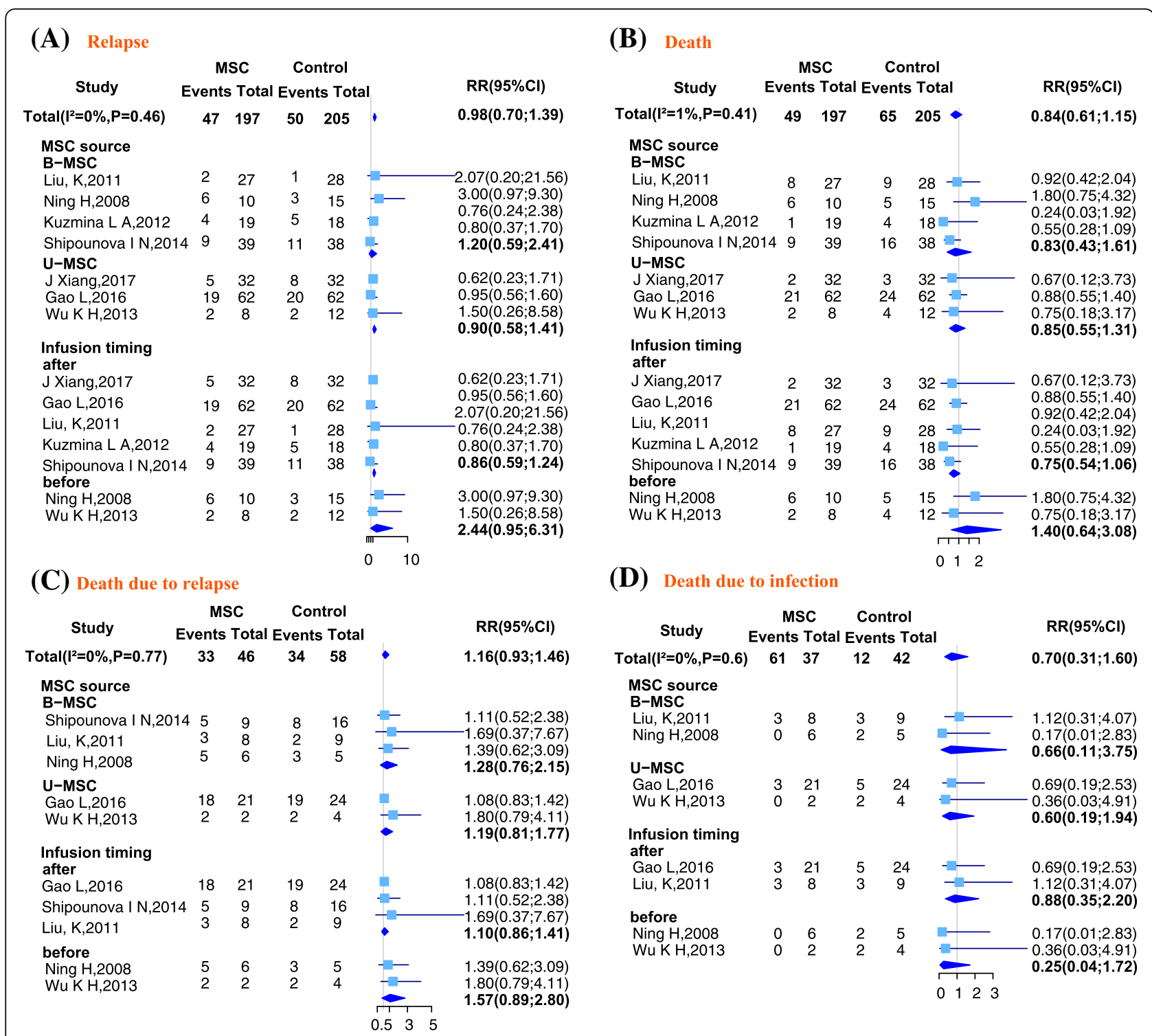

Fig. 4 Forest plot of GVHD treatment: whole and subgroup risk estimates of outcome parameters. a Forest plot showing the overall risk estimate of relapse and the effects of MSC source and infusion timing. b Forest plot showing the overall risk estimate of death and the effects of MSC source and infusion timing. c Forest plot showing the overall risk estimate of death due to relapse and the effects of MSC source and infusion timing. $\mathbf{d}$ Forest plot showing the overall risk estimate of death due to infection and the effects of MSC source and infusion timing

\section{Death}

Seven studies reported the number of patients who died for any reason after HSCT in both the MSC and control groups. Compared with the control group, patients in the MSC group had a lower risk of mortality, but the difference was not statistically significant $(R R=0.84 ; 95 \%$ CI $0.61,1.15 ; I^{2}=1 \%, P=0.41$ ). According to a subgroup analysis based on the source of MSC, both the B-MSC $(\mathrm{RR}=0.83 ; 95 \% \mathrm{CI} 0.43,1.61)$ and $\mathrm{U}-\mathrm{MSC}(\mathrm{RR}=0.85$; $95 \%$ CI $0.55,1.31)$ subgroups showed a non-significant lower risk of mortality compared with the control group. According to subgroup analysis based on MSC infusion time, the after subgroup ( $R R=0.75 ; 95 \%$ CI $0.54,1.06)$ showed a lower (but not statistically significant) risk of death compared with the control group, whereas the before subgroup ( $R R=1.40 ; 95 \%$ CI $0.64,3.08$ ) showed a higher (but not statistically significant) risk of death. These results are shown in Fig. 4.

\section{Death due to relapse}

Five studies reported the number of patients who died after relapse in both the MSC and control groups. Compared with the control group, patients in the MSC group had a higher risk of death due to relapse, but the difference was not statistically significant $(\mathrm{RR}=1.16 ; 95 \% \mathrm{CI}$ $0.93,1.46 ; I^{2}=0 \%, P=0.77$ ). According to a subgroup 
analysis based on the source of MSC, both the B-MSC $(\mathrm{RR}=1.28 ; 95 \% \mathrm{CI} 0.76,2.15)$ and U-MSC subgroups $(\mathrm{RR}=1.19 ; 95 \% \mathrm{CI} 0.81,1.77)$ showed a higher (but not statistically significant) risk of death due to relapse compared with the control group. A subgroup analysis based on MSC infusion time also showed that both the before $(\mathrm{RR}=1.57 ; 95 \% \mathrm{CI} 0.89,2.80)$ and after $(\mathrm{RR}=1.10 ; 95 \% \mathrm{CI}$ $0.86,1.41$ ) subgroups had a higher (but not statistically significant) risk of death due to relapse compared with the control group. These results are shown in Fig. 4.

\section{Death due to infection}

Four studies reported the number of patients who died as a result of infection in both the MSC and control groups. Compared with the control group, patients in the MSC group had a lower risk of death due to infection, but the difference was not statistically significant $\left(\mathrm{RR}=0.70 ; 95 \% \mathrm{CI} 0.31,1.60 ; I^{2}=0 \%, P=0.61\right)$. According to a subgroup analysis based on the source of MSC, both the B-MSC (RR $=0.65$; 95\% CI $0.11,3.75)$ and UMSC ( $R R=0.60 ; 95 \%$ CI $0.19,1.94)$ subgroups showed a lower (but not statistically significant) risk of death due to infection compared with the control group. Subgroup analysis based on MSC infusion time also showed that both the before ( $R R=0.25$; 95\% CI $0.04,1.72$ ) and after $(\mathrm{RR}=0.88 ; 95 \%$ CI $0.35,2.20)$ subgroups had a lower but not statistically significant risk of death caused by infection compared with the control group. These results are shown in Fig. 4.

\section{Discussion}

In this study, we identified, evaluated, and summarized the findings of relevant clinical studies to estimate the efficacy of MSC infusion as a GVHD treatment (one RCT and two nRCTs) and for GVHD prevention (seven RCTs). The findings of our meta-analyses suggest that MSC infusion can reduce cGVHD incidence, but not aGVHD incidence, and has a positive effect on patients who already have aGVHD by increasing the CR rate and prolonging survival.

An increasing number of researchers are noting differences in therapeutic outcomes of MSC treatment among GVHD populations. The use of MSCs for treating aGVHD has received extensive attention, while their use in cases of cGVHD has rarely been reported. In the three trials included in our analysis, only aGVHD was investigated. One possible explanation for this is that the responsiveness of patients with aGVHD to MSCs is superior to that of patients with cGVHD [35]. Since the number of controlled trials investigating MSCs as a treatment for GVHD is limited, we only included three trials. Regardless, our review is the first to evaluate MSC treatment for GVHD in controlled trials and has verified the results of a previous meta-analysis [2] of uncontrolled studies with a single-arm design.

Compared with the treatment of GVHD, controlled trials applying MSCs for GVHD prevention have been widely published. To ensure the reliability of our research, we included only RCTs $(n=7)$ in our analysis. To assess the safety of MSC infusion for GVHD prevention, we performed analyses on the outcomes of engraftment, relapse, and death. Whether or not MSCs increase the incidence of tumor recurrence remains a controversial topic. In fact, MSCs exert bidirectional effects on tumor regulation. On the one hand, MSCs might promote tumor growth and progression, as they can secrete angiogenesispromoting and suppress immune response-suppressing substances [24]; on the other hand, MSCs may inhibit tumors by activating tumor suppression signaling pathways [40]. Despite some studies showing MSCs increased the risk of tumor relapse $[24,41]$, our results showed that MSC infusion had no significant effect on the incidence of relapse, death, or time to neutrophil engraftment.

Currently, one of the most successful clinical applications of MSC infusion is involved in HSCT [42]. However, treatment efficacy varies among clinical trials, and several factors might influence this. To identify factors related to the efficacy of MSC infusion, we conducted subgroup meta-analyses for GVHD prevention according to MSC source and MSC infusion timing. MSCs are readily available from a variety of tissues, including bone marrow, umbilical cord blood, adipose tissue, and the placenta. The studies included in this meta-analysis used only B-MSC or U-MSC, probably because these are the MSC types used most commonly in the clinic. Just as the incidence of GVHD can differ according to the use of stem cells derived from bone marrow versus cord blood units [43], the incidence of GVHD also differs according to use of U-MSCs versus B-MSCs. MSC infusion is advantageous for reducing cGVHD incidence, but this was seen only in the U-MSC subgroup and not the B-MSC subgroup in this study. Although MSCs had no overall significant effect on the time to neutrophil engraftment, a significant improvement was seen in the U-MSC subgroup. Regarding relapse, despite no significant difference between the overall metaanalysis and subgroup meta-analysis, use of U-MSCs tended to reduce relapse, whereas use of B-MSCs tended to increase relapse. These findings suggest that B-MSCs are not a good candidate cell type for GVHD prophylaxis in comparison with U-MSCs.

Although adult bone marrow has served as the traditional source of MSCs, fetal-type MSCs, such as UMSCs, have also proven to be an excellent alternative source [44]. These MSCs can be obtained more easily, proliferate faster in vitro, and show reduced 
immunogenicity [45-47]. More importantly, compared with adult-type MSCs, fetal-type MSCs have stronger immunosuppressive effects [48]. The reduced immunogenicity and stronger immunosuppressive effects of UMSCs make them ideal candidates for cell-based therapies, especially for diseases associated with an immune response [48]. The use of U-MSCs for reducing cGVHD incidence and promoting engraftment shows much promise, and further clinical studies involving fetal-type MSCs, such as U-MSCs, in HSCT are urgently needed.

Infusion timing, of which little is known, is another important factor when evaluating the efficacy of MSC-based therapy $[49,50]$. Our subgroup analysis of infusion timing revealed that MSC infusion after HSCT had a greater beneficial effect, with significant improvements seen in engraftment and a lower risk of cGVHD incidence occurring only in the after, and not the before, subgroup. In addition, despite a lack of statistical significance, MSC infusion after HSCT tended to reduce the incidence rates of relapse and death, whereas MSC infusion before HSCT tended to increase the incidence rates. The influence of timing of MSC infusion might be related to differences in the immune and inflammation microenvironment in vivo over time [51].

CGVHD is the leading cause of mortality and morbidity after HSCT. Given that our findings showed that MSC infusion decreased cGVHD incidence, its inability to prolong survival seems to be inexplicable and warrants further study. In addition to cGVHD, relapse and infection are the other major causes of death after HSCT [52]. We therefore conducted analyses to determine how MSC infusion affected death due to relapse and death. The results showed that MSC infusion may increase the likelihood of death due to relapse after HSCT. Although the increase was not statistically significant, it remains a concern. Along with the findings showing that the use of B-MSC infusion, and MSC infusion before HSCT, also tended to increase the risk of relapse, MSCs for GVHD prevention failed to enhance survival, which may be related to a higher incidence of relapse and death due to relapse.

There were several limitations to this systematic review. First, we could not precisely estimate the overall treatment efficacy because of the small number of studies and their small sample sizes, especially with respect to GVHD treatment. Although we included both RCTs and nRCTs on GVHD treatment, there were insufficient studies and patients to perform a subgroup meta-analysis. Our findings could be further validated or refined by integrating other data, provided additional relevant literature is published soon. In addition, we were unable to assess publication bias, either statistically or visually, because of the limited number of included studies. Second, possibly because of the small number of studies and their small sample sizes, the statistical power was limited. The possible advantages of UMSCs over B-MSCs, and of MSC infusion after HSCT versus before HSCT, in terms of decreasing relapse or death, were not statistically demonstrated. Therefore, these results require further validation via additional research. The possible disadvantages of B-MSCs and MSC infusion before HSCT also need to be verified to avoid harming patients. In addition, the possibility that MSC infusion may increase the likelihood of death due to relapse also needs to be further explored. Third, we were unable to assess the effects of some important parameters such as the recipient age, type of MSC donor, type of cancer being treated, preparative therapy before transplantation, and role of HLA mismatching which all could influence the clinical outcomes. In our initial study design, subgroup analyses were to be performed if all seven studies reported the information required and the data could be stratified. Unfortunately however, subgroup analyses based on these variables could not be performed because of an inadequate number of studies or relevant data. These limitations introduced unreliability into our study, but our work should still be interesting to researchers and clinicians devoted to use of MSCs as a safe and effective approach for GVHD.

\section{Conclusion}

In conclusion, the evidence from RCTs and nRCTs suggests that MSCs can play a useful role in HSCT, including by promoting engraftment (U-MSC), preventing GVHD (mainly cGVHD), and ameliorating GVHD (aGVHD). MSC infusion enhanced survival only in the context of treating, and not preventing GVHD. For GVHD prevention, use of U-MSCs and infusion after HSCT were optimal for suppressing cGVHD incidence and promoting engraftment and may decrease the incidence rates of relapse and death. The use of B-MSCs and infusion before HSCT may be harmful to the patient and thus requires serious consideration. In closing, these findings need further confirmation as the limitations imposed on this meta-analysis by the small number of included studies and their small number of patients. Therefore, future studies need to determine the clinical impact of MSC infusion for treating GVHD, and our research needs to be validated via a sufficient number of high-quality clinical trials with large numbers of patients.

\section{Additional file}

Additional file 1: Search strategy. (PDF $12 \mathrm{~kb}$ ) 


\section{Abbreviations}

aGVHD: Acute graft-versus-host disease; ALG: Antilymphocyte globulin; ATG: Antithymocyte globulin; B-MSC: MSC derived from the bone marrow; CGVHD: Chronic graft-versus-host disease; Cl: Confidence intervals;

CR: Complete response; GVHD: Graft-versus-host disease;

HSCT: Hematopoietic stem cell transplantation; MSC: Mesenchymal stem cells; nRCTs: Non-randomized controlled trials; RCT: Randomized controlled trials.; RR: Risk ratio; SMD: Standardized mean difference; U-MSC: MSC derived from the umbilical cord

\section{Acknowledgements \\ Not applicable.}

\section{Authors' contributions}

HC designed the study. LZ, SC, and PY abstracted the data, performed the analysis, and wrote the paper. LL supervised the study. All authors reviewed and approved the final version of the manuscript.

\section{Funding}

This study was supported by the National Key Research and Development Program of China (No. 2016YFA0101001) and National Natural Science Foundation of China (No. 81620108028).

\section{Availability of data and materials}

All supporting data are included in the article and its Additional file 1.

\section{Ethics approval and consent to participate}

Not applicable.

\section{Consent for publication}

Not applicable.

\section{Competing interests}

The authors declare that they have no competing interests.

\section{Author details}

${ }^{1}$ State Key Laboratory for Diagnosis and Treatment of Infectious Diseases, the First Affiliated Hospital, College of Medicine, Zhejiang University, 79 Qingchun Rd., Hangzhou City 310003, China. ${ }^{2}$ Collaborative Innovation Center for Diagnosis and Treatment of Infectious Diseases, 79 Qingchun Rd., Hangzhou City 310003 , China. ${ }^{3}$ The School of Clinical Medicine, University of Cambridge, Cambridgeshire, UK.

\section{Published online: 21 June 2019}

\section{References}

1. Kanakry CG, Fuchs EJ, Luznik L. Modern approaches to HLA-haploidentical blood or marrow transplantation. Nat Rev Clin Oncol. 2016;13(2):132.

2. Hashmi S, Ahmed M, Murad MH, et al. Survival after mesenchymal stromal cell therapy in steroid-refractory acute graft-versus-host disease: systematic review and meta-analysis. Lancet Haematol. 2016;3(1):e45-52.

3. Welniak LA, Blazar BR, Murphy WJ. Immunobiology of allogeneic hematopoietic stem cell transplantation. Annu Rev Immunol. 2007;25:139-70

4. Petinaty N, Shipounova I, Kuzmina L, et al. Multipotent mesenchymal stromal cells as graft-versus-host disease prophylaxis: a prospective randomised clinical trial. Blood. 2010;116(21):538.

5. Martin PJ, Rizzo JD, Wingard JR, et al. First- and second-line systemic treatment of acute graft-versus-host disease: recommendations of the American Society of Blood and Marrow Transplantation. Biol Blood Marrow Transplant. 2012;18(8):1150-63.

6. Ferrara JL, Levine JE, Reddy P, Holler E. Graft-versus-host disease. Lancet. 2009;373(9674):1550-61.

7. Van Lint MT, Uderzo C, Locasciulli A, et al. Early treatment of acute graftversus-host disease with high- or low-dose 6-methylprednisolone: a multicenter randomized trial from the Italian Group for Bone Marrow Transplantation. Blood. 1998;92(7):2288-93.

8. Martin PJ, Schoch G, Fisher L, et al. A retrospective analysis of therapy for acute graft-versus-host disease: initial treatment. Blood. 1990;76(8):1464-72.

9. Gao L, Zhang Y, Hu B, et al. Phase II multicenter, randomized, double-blind controlled study of efficacy and safety of umbilical cord-derived mesenchymal stromal cells in the prophylaxis of chronic graft-versus-host disease after HLA-haploidentical stem-cell transplantation. J Clin Oncol. 2016:34(24):2843-50

10. Cahn JY, Klein JP, Lee SJ, et al. Prospective evaluation of 2 acute graftversus-host (GVHD) grading systems: a joint Societe Francaise de Greffe de Moelle et Therapie Cellulaire (SFGM-TC), Dana Farber Cancer Institute (DFCI), and International Bone Marrow Transplant Registry (IBMTR) prospective study. Blood. 2005;106(4):1495-500.

11. Uccelli A, Moretta L, Pistoia V. Immunoregulatory function of mesenchymal stem cells. Eur J Immunol. 2006;36(10):2566-73.

12. Fierabracci A, Del FA, Muraca M, Delfino DV, Muraca M. The use of mesenchymal stem cells for the treatment of autoimmunity: from animals models to human disease. Curr Drug Targets. 2016;17(2):229-38.

13. Dan YY, Riehle KJ, Lazaro C, et al. Isolation of multipotent progenitor cells from human fetal liver capable of differentiating into liver and mesenchymal lineages. Proc Natl Acad Sci U S A. 2006:103(26):9912-7.

14. Bartholomew A, Sturgeon C, Siatskas M, et al. Mesenchymal stem cells suppress lymphocyte proliferation in vitro and prolong skin graft survival in vivo. Exp Hematol. 2002;30(1):42-8.

15. Puissant B, Barreau C, Bourin P, et al. Immunomodulatory effect of human adipose tissue-derived adult stem cells: comparison with bone marrow mesenchymal stem cells. Br J Haematol. 2005;129(1):118-29.

16. Corcione A, Benvenuto F, Ferretti $E$, et al. Human mesenchymal stem cells modulate B-cell functions. Blood. 2006;107(1):367-72.

17. Ramasamy R, Fazekasova H, Lam EW, Soeiro I, Lombardi G, Dazzi F. Mesenchymal stem cells inhibit dendritic cell differentiation and function by preventing entry into the cell cycle. Transplantation. 2007;83(1):71-6.

18. Spaggiari GM, Capobianco A, Becchetti S, Mingari MC, Moretta L. Mesenchymal stem cell-natural killer cell interactions: evidence that activated NK cells are capable of killing MSCs, whereas MSCs can inhibit IL2-induced NK-cell proliferation. Blood. 2006;107(4):1484-90.

19. Dazzi F, Ramasamy R, Glennie S, Jones SP, Roberts I. The role of mesenchymal stem cells in haemopoiesis. Blood Rev. 2006;20(3):161-71.

20. Devine SM, Hoffman R. Role of mesenchymal stem cells in hematopoietic stem cell transplantation. Curr Opin Hematol. 2000;7(6):358-63.

21. Wang S, Qu X, Zhao RC. Clinical applications of mesenchymal stem cells. J Hematol Oncol. 2012;5:19.

22. Le Blanc K, Rasmusson I, Sundberg B, et al. Treatment of severe acute graftversus-host disease with third party haploidentical mesenchymal stem cells. Lancet. 2004;363:1439-41.

23. Galipeau J. The mesenchymal stromal cells dilemma--does a negative phase III trial of random donor mesenchymal stromal cells in steroid-resistant graft-versushost disease represent a death knell or a bump in the road? Cytotherapy. 2013;15:2-8.

24. Ning $\mathrm{H}$, Yang $\mathrm{F}$, Jiang $\mathrm{M}$, et al. The correlation between cotransplantation of mesenchymal stem cells and higher recurrence rate in hematologic malignancy patients: outcome of a pilot clinical study. Leukemia. 2008;22(3):593-9.

25. Liu K, Chen Y, Zeng Y, et al. Coinfusion of mesenchymal stromal cells facilitates platelet recovery without increasing leukemia recurrence in haploidentical hematopoietic stem cell transplantation: a randomized, controlled clinical study. Stem Cells Dev. 2011;20(10):1679-85.

26. PubMed. Available at https://www.ncbi.nlm.nih.gov/pubmed. Accessed 27 Feb 2018.

27. Cochrane Library. Available at http://www.cochranelibrary.com/. Accessed 27 Feb 2018.

28. Embase. Available at https://www.embase.com/\#search. Accessed 27 Feb 2018.

29. ClinicalTrials.gov. Available at https://clinicaltrials.gov/. Accessed 27 Feb 2018.

30. Chinese Biological and Medical Databasev (Sinomed). Available at http:// www.sinomed.ac.cn/cross/advancedSearch.html . Accessed 27 Feb 2018.

31. Egger M, Davey SG, Schneider M, Minder C. Bias in meta-analysis detected by a simple, graphical test. BMJ. 1997;315(7109):629-34.

32. Martin PJ, Uberti JP, Soiffer RJ, et al. Prochymal improves response rates in patients with steroid-refractory acute graft versus host disease (SR-GVHD) involving the liver and gut: results of a randomized, placebo-controlled, multicenter phase III trial in GVHD. Biol Blood Marrow Transplant. 2010;16(2, Supplement 2):S169-70.

33. Xiang J. Effect of human umbilical cord mesenchymal stem cells on immune reconstruction of acute lymphoblastic leukemia children undergoing allogeneic hematopoietic stem cell transplantation. Chin J Tissue Eng Res. 2017;21(29):4679-84 in Chinese. 
34. Shipounova IN, Petinati NA, Bigildeev AE, et al. Analysis of results of acute graft-versus-host disease prophylaxis with donor multipotent mesenchymal stromal cells in patients with hemoblastoses after allogeneic bone marrow transplantation. Biochemistry (Mosc). 2014;79(12):1363-70.

35. Kuzmina LA, Petinati NA, Parovichnikova EN, et al. Multipotent mesenchymal stromal cells for the prophylaxis of acute graft-versus-host disease-a phase II study. Stem Cells Int. 2012(1):968213.

36. Wu KH, Tsai C, Wu HP, Sieber M, Peng CT, Chao YH. Human application of ex vivo expanded umbilical cord-derived mesenchymal stem cells: enhance hematopoiesis after cord blood transplantation. Cell Transplant. 2013;22(11): 2041-51.

37. Zhao K, Lou R, Huang F, et al. Immunomodulation effects of mesenchymal stromal cells on acute graft-versus-host disease after hematopoietic stem cell transplantation. Biol Blood Marrow Transplant. 2015;21(1):97-104.

38. Remberger $\mathrm{M}$, Ringden $\mathrm{O}$. Treatment of severe acute graft-versus-host disease with mesenchymal stromal cells: a comparison with non-MSC treated patients. Int J Hematol. 2012;96(6):822-4.

39. Szabolcs P, Visani G, Locatelli F, et al. Treatment of steroid-refractory acute GvHD with mesenchymal stem cells improves outcomes in paediatric patients. Results of the paediatric subset in a phase III randomized, placebocontrolled study. Bone Marrow Transplant. 2010;(Suppl 2s):S18.

40. Wu K, Wu H, Chan C, Hwang S, Peng C, Chao Y. The role of mesenchymal stem cells in hematopoietic stem cell transplantation: from bench to bedsides. Cell Transplant. 2013;22(4):723-9.

41. Sun Z, Wang S, Zhao RC. The roles of mesenchymal stem cells in tumor inflammatory microenvironment. J Hematol Oncol. 2014;7:14.

42. Kogler G, Radke TF, Lefort A, et al. Cytokine production and hematopoiesis supporting activity of cord blood-derived unrestricted somatic stem cells. Exp Hematol. 2005;33(5):573-83.

43. Kim EJ, Kim N, Cho SG. The potential use of mesenchymal stem cells in hematopoietic stem cell transplantation. Exp Mol Med. 2013;45:e2.

44. Anasetti C, Logan BR, Lee SJ, et al. Peripheral-blood stem cells versus bone marrow from unrelated donors. N Engl J Med. 2012;367(16):1487-96.

45. Zhang ZY, Teoh SH, Chong MS, et al. Superior osteogenic capacity for bone tissue engineering of fetal compared with perinatal and adult mesenchymal stem cells. Stem Cells. 2009;27(1):126-37.

46. Lu LL, Liu YJ, Yang SG, et al. Isolation and characterization of human umbilical cord mesenchymal stem cells with hematopoiesis-supportive function and other potentials. Haematologica. 2006;91(8):1017-26.

47. Secco M, Zucconi E, Vieira NM, et al. Multipotent stem cells from umbilical cord: cord is richer than blood! Stem Cells. 2008;26(1):146-50.

48. Chan CK, Wu KH, Lee YS, et al. The comparison of interleukin 6-associated immunosuppressive effects of human ESCS, fetal-type MSCs, and adult-type MSCs. Transplantation. 2012;94(2):132-8.

49. Locatelli F, Algeri M, Trevisan V, Bertaina A. Remestemcel-L for the treatment of graft versus host disease. Expert Rev Clin Immunol. 2017; 13(1):43-56.

50. Griffin TP, Martin WP, Islam N, O'Brien T, Griffin MD. The promise of mesenchymal stem cell therapy for diabetic kidney disease. Curr Diab Rep. 2016;16(5):42.

51. Te BL, Mansilla C, van der Wagen LE, et al. Biomarker profiling of steroidresistant acute GVHD in patients after infusion of mesenchymal stromal cells. Leukemia. 2015;29(9):1839-46.

52. Wingard JR, Majhail NS, Brazauskas R, et al. Long-term survival and late deaths after allogeneic hematopoietic cell transplantation. J Clin Oncol. 2011;29(16):2230-9.

\section{Publisher's Note}

Springer Nature remains neutral with regard to jurisdictional claims in published maps and institutional affiliations. 